\title{
ANÁLISIS DE LA DIFUSIÓN DEL ACEITE ESENCIAL DE MUÑA (Clinopodium bolivianum) EN VAPOR DE AGUA
}

\author{
ANALYSIS OF THE DIFFUSION OF MUÑA'S (Clinopodium bolivianum) \\ ESSENTIAL OIL INTO WATER STEAM
}

\author{
'Vicente Málaga Apaza
}

\begin{abstract}
RESUMEN
Se estudió el efecto de sustratos vegetales sobre el rendimiento, difusión y velocidad de extracción de aceites esenciales, usando vapor de agua. Como sustratos vegetales se utilizaron hojas y tallos de muña (Clinopodium bolivianum). La presente memoria establece nuevas metodologias para comprender la transferencia de masa, mediante un modelado matemático básico, versátil y consistente. Ubicar el aceite esencial en la matriz herbácea, mediante el análisis micro-histológico por SEM y se determinó la reologia del aceite esencial; a fin de ofrecer criterios para el diseño de nuevos extractores. Se procesaron una variedad representativa de dos matrices herbáceas conocidas: hojas y tallos, en un equipo bench, con diferentes flujos del vapor de agua y diferentes porosidades del lecho vegetal. Se obtuvo exitosamente sus aceites esenciales y se caracterizó positivamente según la normativa técnica vigente y según estudios previos publicados, mediante, refractometrí, picnometria y reologia. La planta en estudio fue la muña (Clinopodium bolivianum), con un rendimiento de 1,362\%, en 12 pruebas experimentales, con una carga fija por prueba; usando sus hojas y tallos parcialmente cegados. Se determinó el tipo de fluido del aceite esencial más la viscosidad, que en este caso es viscoplástico correspondiendo a un modelo tipo CASSON, viscosidad a $13^{\circ} \mathrm{C}$ es de $3,8 \mathrm{cP}$ y a $30^{\circ} \mathrm{C}$ es de $2,8 \mathrm{cP}$. El modelado fenomenológico desarrollado predice la evolución de la hidrodestilación con una precisión aceptable. Los fenómenos controlantes del proceso son la exudación térmica del aceite esencial desde los tricomas glandulares, la evaporación instantánea del aceite en la interfase de la pelicula superficial y la corriente de vapor circundante, y la difusión por convección del aceite en la corriente de vapor que atraviesa el lecho vegetal. Los parámetros del modelado fueron la concentración de aceite y la constante de velocidad de la exudación térmica $(K)$, que sirven para comparar la rapidez de la difusión efectiva del aceite esencial de una planta frente a otra; y el área de transferencia de masa efectiva entre distintas matrices herbáceas. Mediante el análisis micro-histológico, se determinó que el aceite esencial está situado en los tricomas glandulares superficiales de las flores y hojas de vegetales estudiadas. En el caso de la muña, los tricomas glandulares se encuentran en ambos lados de las hojas.
\end{abstract}

Palabras Clave: aceite esencial, extracción, difusión, muña, vapor de agua, velocidad

\section{ABSTRACT}

In this paper the effect of plant substrata on the yield. diffusion and speed of extraction of essential oils using water steam has been studied. As vegetable substrata, muña's leaves and stems were used (Clinopodium bolivianum). This memory states new methodology to: understand mass transfer through a basic, versatile and consistent mathematical modeling: locate the essential oil in the herbaceous matrix by micro-histological analysis by SEM. The rheology of the essential oil was determined to offer new criteria to design new extractors. A representative variety of two well-known herbaceous matrices was processed: leaves and stems in bench equipment with different water steam flows and different vegetable bed porosities. The essential oils were obtained successfully and they were also positively characterized according to the technical in force regulations or previous published studies by refractometry, pycnometry and rheology. The plant under study was commonly referred as muna (Clinopodium bolivianum) yielding $1.362 \%$ in 12 experimental test, with a fixed charge per test by using their leaves and stems partially blinded. The fluid type of essential oil plus the viscosity were determined which in this case the viscosity is viscoplastic that responds to a model type CASSON viscosity at 13 degrees Celsius is $3.8 \mathrm{cP}$ and at 30 degrees Celsius is $2.8 \mathrm{cP}$. The developed phenomenological modeling predicts the hydrodistillation evolution with acceptable precision. The controllable phenomena of the process are thermal exudation of the essential oil from the glandular trichomes, the instantaneous evaporation of oil at the interface of the surface film, the surrounding current steam, the diffusion by convection of oil in steam current that crosses the vegetable bed. The modeling parameters: the concentration of oil, the speed constant of thermal exudation $(K)$; they are helpful to compare the effective diffusion speed of the essential oil from a plant with another, and the area of effective mass transfer among different herbaceous matrices. By means of micro-histological analysis, it was determined that the essential oil is located in the superficial glandular trichomes of the studied flowers and leaves. In the case of the muña, glandular trichomes are located on
both sides of the leaves.

Key Words: essential oil, mining, broadcasting, muña, steam, speed 


\section{I.INTRODUCCIÓN}

En los últimos años, varios trabajos de investigación han sido publicados, proponiendo nuevos enunciados matemáticos basados en el balance microscópico del transporte del aceite en el vapor, que explicarían los fenómenos mencionados en la metodología.

Palomino y Cerpa (1999) aplicaron su modelado al caso de las hojas de la muña (Minthostachys Setosa L.) consideran que la exudación es una difusión intraparticula del aceite esencial en el vapor penetrante de la matriz herbácea. La vaporización del aceite liberado se asume como un fenómeno cinético, representado por una velocidad de primer orden con respecto a la concentración en el equilibrio del aceite vaporizado en el vapor de agua. Relaciona la vaporización con la difusión intrapartícula, a través de un factor de efectividad de extracción, análogo al módulo de Thiele, usado para la reacción heterogénea en un sólido poroso. Se despreció la difusión externa por convección y sólo los procesos internos controlaban el proceso. Este modelado posee algunas limitaciones en su fundamento, uno es que el aceite esencial se encuentra mayoritariamente en los tricomas glandulares superficiales y no en el interior de las hojas; la vaporización instantánea depende del equilibrio de las fases formadas y no es un término arbitrario o empírico. Además, la aplicación de un factor de efectividad es inconsistente físicamente, debido a que el soluto es liberado desde la superficie de la matriz hacia el seno del vapor y el módulo de Thiele es aplicado cuando el soluto penetra en el sólido.

Benyoussef et al., (2002) aplicaron su modelado al caso de los frutos del cilantro (Coriandum sativum L.). Consideró que la exudación es una desorción irreversible del aceite esencial en el agua, penetrante en la matriz herbácea, con una velocidad de primer orden, respecto al aceite retenido. La vaporización del aceite liberado se asumió como instantánea, debido al equilibrio de fases formado, pero la relación era explícita y en función de un término constante y empírico. La difusión intrapartícula del aceite en el agua penetrante fue asumida como la controlante, despreciándose la difusión externa por convección. Este modelado posee algunas limitaciones en su enunciado, sólo es aplicable a frutos (geometría esferoide), donde el aceite esencial está en el interior de la matriz herbácea; la difusión intrapartícula del aceite en el vapor penetrante se aplica sólo cuando la materia prima está molida, como fue el caso, pero a nivel industrial, los frutos no suelen ser molidos y no hay evidencias de que el agua penetre en la matriz herbácea; el término empírico para relacionar el equilibrio entre el aceite en la superficie de la matriz y el del vapor es definido como un coeficiente de reparto, independiente de las volatilidades $y$ de la solubilidad del aceite en el vapor, $y$ sin ninguna relación termodinámica que lo respalde; lo cual es inconsistente, porque el aceite esencial se vaporiza en determinada proporción, de acuerdo a principios termodinámicos conocidos.

Romdhane y Tizaoui (2005) aplicó un su modelado al caso de los frutos del anís verde (pimpinella anisum). Considera que no existe exudación; el aceite es transportado desde el interior de los frutos por un gradiente de concentraciones y la velocidad de transferencia depende de ese gradiente y de un coeficiente global. La vaporización del aceite liberado se asume como instantánea, debido al equilibrio de fases formado, pero la relación era explícita y en función de un término constante y empírico, junto con la presión de vapor del compuesto químico mayoritario en el aceite. Este modelado posee algunas limitaciones en su enunciado, pues sólo es aplicable a frutos (geometría esferoide), donde el aceite esencial está en el interior de la matriz herbácea; el término empírico para relacionar el equilibrio entre el aceite en la superficie de la matriz y el del vapor es definido como un parámetro de proporcionalidad, independiente de las volatilidades $y$ de la solubilidad del aceite en el vapor, asumiéndose un similitud con la constante de Henry. Sin embargo, este parámetro es calculado por la minimización de la función objetivo y sin el uso de las propiedades físicas de los compuestos químicos involucrados, ni de relaciones termodinámicas conocidas. La disminución de aceite esencial en la matriz herbácea se calcula mediante un balance global de materia y en función de la velocidad de transferencia debido a la difusión por convección.

De la anterior compilación de modelados publicados, se deduce que la difusión intrapartícula, llámese de aquella que ocurre dentro de la matriz herbácea, controla el proceso, con la excepción del trabajo de Sovová y Aleksovski (2006). El equilibrio entre el aceite esencial superficial de la matriz y el vaporizado en la corriente es expresado como un coeficiente de partición, independiente de la termodinámica de fases y obtenido por la minimización de la función objetivo del modelado, con respecto a los datos experimentales. La exudación es un fenómeno intrapartícula y puede ser expresada como una desorción irreversible de primer orden. Estas hipótesis contradicen 3 evidencias reales: la variación del flujo del vapor usado afecta directamente la velocidad de obtención del aceite esencial; el equilibrio entre el aceite liquido superficial y el vaporizado debe obedecer los principios generales de la termodinámica de fases y no deberia ser relacionado empíricamente; la exudación no es un fenómeno interno, sino externo (superficial), que afectará de diferente manera al proceso, cuando la materia prima está intacta o triturada. A nivel industrial, la materia prima está intacta o parcialmente reducida, se trabaja con diferentes caudales de vapor para determinar el óptimo, técnica y económicamente. Por lo tanto, existe la necesidad, de formular nuevos modelados fenomenológicos, capaces de representar matemáticamente los tres fenómenos enunciados previamente, que sean consistentes físicamente con los hallazgos realizados por el micro histologia.

\section{Formulación del modelo matemático de difusión}

$$
\begin{aligned}
\frac{\partial C_{A}}{\partial t} & =D_{A B}\left(\frac{\partial^{2} C_{A}}{\partial x^{2}}+\frac{\partial^{2} C_{A}}{\partial y^{2}}+\frac{\partial^{2} C_{A}}{\partial z^{2}}\right) \\
\rho_{0} \frac{\partial x}{\partial t} & =\nabla \cdot\left(-J_{m}\right)
\end{aligned}
$$

Por medios isótropos, los flujos de masa se expresan mediante:

$$
J_{m}=-\lambda_{m q} \nabla T-a_{m} \rho_{0} \nabla X
$$


Para sólidos aniso trópicos, estas expresiones pueden ser generalizadas:

$$
J_{m x_{i}}=-\sum_{j} a_{m_{i j}} \rho_{0} \frac{\partial X}{\partial x_{j}}
$$

Donde fueron eliminados los términos correspondientes a los efectos Duffor y Soret ya que $\lambda_{\text {mq }}$ son despreciables en los procesos considerados.

La cantidad $\mathrm{a}_{\mathrm{mil}}$, son los componentes de un tensor de segundo orden. Cuando el sólido presenta algún tipo de simetría, las expresiones anteriores pueden simplificarse a través de la elección apropiada del sistema de ejes coordenados. Condiciones iniciales y de contorno. Del balance de masa se tiene:

$$
\left.a_{m} \rho_{0} \frac{\partial x}{\partial n}\right|_{S}=\alpha_{m} \Delta P
$$

$a_{m}$ es el coeficiente de transferencia de masa por convección debido a la diferencia de presiones parciales de vapor $(\Delta \mathrm{P})$ entre el aire adyacente a la superficie y el medio ambiente. La presión parcial de aire $\mathrm{f}(\mathrm{T})$ se calcula en función de su temperatura y su humedad relativa. Para la evaluación de la presión de vapor sobre la superficie, se considera que, instantáneamente, la superficie se encuentra en equilibrio con el vapor adyacente, ambos a la temperatura $\mathrm{T}_{\mathrm{s}}$. Por lo tanto:

$$
\Delta P=f\left(T_{S}\right)-f\left(T_{a}\right)
$$

\section{Aplicación a la difusión del aceite esencial en vapor de} agua.

Las hojas de muña se comportan como material aniso trópico debido a la presencia rugosa en la superficie de su estructura. Para su estudio es conveniente elegir una tableta plana rugosa como dominio y orientar un sistema de placa plana en una dirección $z$. De esta forma el tensor $a_{\text {min }}$ sólo presenta un componente no nulo, correspondiente a las fibras de la hoja y normal a las mismas. Aplicando la divergencia a la ecuación (3) se tiene:

$$
-\Delta\left(J_{m}\right)=\frac{a_{m x}}{x} \rho_{0} \frac{\partial^{2} x}{\partial x^{2}}+a_{m z} \rho_{0} \frac{\partial^{2} T}{\partial Z^{2}}
$$

Esquema simplificado para el análisis:

$$
\frac{\partial X}{\partial t}=a_{m} \frac{\partial^{2} X}{\partial x^{2}}
$$

Ahora podemos expresar el problema completo, en función de $\theta(z, t)$ como:

$$
\mathrm{D}_{\mathrm{Ac}} \frac{\partial^{2} \theta}{\partial z^{2}}=\frac{\partial \theta}{\partial t}
$$

Luego aplicando la técnica usual de separación de variable haciendo.

$$
\theta(z, t)=X(z) \cdot \tau(t)
$$

Donde $\mathrm{X}(\mathrm{z})$ y $\tau(\mathrm{t})$ son función únicamente de los parámetros z y t respectivamente.

Sustituyendo (10) en (9)

Málaga V., Anàlisis de la difusión del aceíte esencial de muña (Clinopodium bolivianum) en vapor de agu

$$
1 / \mathrm{D}_{A_{\varepsilon}} \frac{1}{\tau} \frac{d \tau}{d t}=\frac{1}{\mathrm{X}} \frac{d^{2} \mathrm{X}}{d z^{2}}
$$

Cada lado de la ecuación 11 es una función solamente de una variable independiente; por tanto ambos deben ser iguales a alguna constante, que se puede llamar $\lambda^{2}$. Igualando cada lado a $\lambda^{2}$ a la vez, se tiene dos ecuaciones diferenciales ordinarias.

$$
\begin{aligned}
& \frac{d \tau}{d t}=\mathrm{D}_{\mathrm{ac}} \tau \lambda^{2} \\
& \frac{d^{2} X}{d z^{2}}=z \lambda^{2}
\end{aligned}
$$

Indica que $\tau$ es una función rápidamente creciente de $t$, que se aproxima al infinito para grandes valores de $t$, desde luego, fisicamente es una situación imposible; por tanto, la constante $\lambda^{2}$ debe tener un signo negativo y las ecuaciones diferenciales ordinarias a resolver son:

$$
\begin{aligned}
\frac{d \tau}{\tau} & =-\mathrm{D} \tau \lambda^{2} \\
\frac{d^{2} X}{d z^{2}} & =-\lambda^{2} Z
\end{aligned}
$$

Resolviendo para (14) y (15) se tiene:

$$
\frac{d \tau}{\tau}=-\mathrm{D} \lambda^{2} d t
$$

Ahora la expresión completa para $\theta$ queda:

$$
\theta(z, t)=\tau(t) \cdot X(z)=A e^{-D \lambda^{2} t} \cdot[B \cos \lambda z+C \operatorname{sen}-\lambda z]
$$

Para que la expresión sea verdadera, la constante $\mathrm{N}$ debe ser igual a cero. La expresión restante para $\theta(z, t)$ es:

$$
\begin{aligned}
& \frac{\partial \theta}{\partial z}(z, t)=M e^{-D \lambda^{2} t} \cdot \cos z \\
& \operatorname{tg} \lambda L=\frac{h}{D \lambda}
\end{aligned}
$$

Si se multiplica y divide el lado derecho de esta ecuación por $\mathrm{L}$ la razón $\mathrm{L} / \lambda \mathrm{D}$ queda:

$$
\operatorname{tg} \lambda L=\frac{B i}{\lambda L}
$$

Se denota por $\lambda_{n}, \lambda_{y} \ldots \lambda_{n}$, cada valor de $\lambda$ que satisface la ecuación (19), en donde $1 \leq n<\infty$, y la solución es de la forma:

$$
\theta_{n}(z, t)=M_{n} e^{-D \lambda_{n}{ }^{2} t} \cdot \cos \lambda_{n} z
$$

Ahora se puede eliminar el signo de la sumatoria, ya que el lado derecho de esta expresión desaparece para valores $\mathrm{de} \mathrm{m} \neq \mathrm{n}$.

$$
M_{n}=\frac{2 \operatorname{sen} \lambda_{n} z}{\lambda_{n} L+\operatorname{sen} \lambda_{n} L \cdot \cos \lambda_{n} L}
$$

Con lo que en seguida se puede escribir la solución final para $\theta(z, t)$ como: 


$$
\frac{\theta(z, t)}{\theta_{o}}=2 \sum_{n=1}^{\infty} e^{-D \lambda^{2} t} \frac{\operatorname{sen} \lambda_{n} L \cdot \cos \lambda_{n} L}{\lambda_{n} L+\operatorname{sen} \lambda_{n} L \cdot \cos \lambda_{n} L}
$$

$\mathrm{O} \operatorname{con} \delta_{\mathrm{n}}=\lambda_{\mathrm{n}} \mathrm{L}$, se puede escribir la ecuación 23 en la forma:

$$
\frac{\theta(z, t)}{\theta_{o}}=2 \sum_{n=1}^{\infty} e^{-\delta_{n}^{2}\left(D t / L^{2}\right)} \frac{\operatorname{sen} \delta_{n} \cdot \cos \delta_{n} z / L}{\delta_{n}+\operatorname{sen} \delta_{n} \cdot \cos \delta_{n}}
$$

\section{MATERIALES Y MÉTODOS}

Los fundamentos de la destilación por arrastre con vapor de agua, consisten en utilizar, para separar sustancias ligeramente volátiles e insolubles en agua, otros productos no volátiles mezclados con ellas. Para la comprensión de ésta operación, se hace la consideración del comportamiento en la destilación de un sistema de dos fases formado por dos líquidos, x e y, completamente insolubles entre sí (agua y aceite esencial); cada líquido ejerce su propia tensión de vapor, independiente de la otra. Así, la Presión total $\left(\mathrm{P}_{\mathrm{T}}\right)$, se puede calcular de la siguiente forma:

$$
P_{T}=P_{X}+P_{Y} \quad(\text { a T })
$$

Siendo:

$\mathrm{P}_{\mathrm{X}}=$ Presión de vapor de $\mathrm{X}$ a $\mathrm{T}$

$\mathrm{P}_{\mathrm{Y}}=$ Presión de vapor de $\mathrm{YaT}$

El punto de ebullición de la mezcla será aquella temperatura en la que la presión total $\mathrm{P}_{\mathrm{T}}$, sea igual a la atmosférica. Puesto que la presión ejercida por un gas (a una temperatura dada) es proporcional a la concentración de sus moléculas, la relación de las presiones de vapor de X e Y, en el punto de ebullición de la mezcla será igual a la relación entre el número de moléculas de $\mathrm{X}$ y de $\mathrm{Y}$ que destilan de la mezcla. Así la composición del vapor se puede calcular de la siguiente forma:

$$
\frac{N_{X}}{N_{T}}=\frac{P_{X}}{P_{T}}
$$

Siendo:

$\mathrm{N}_{\mathrm{x}} / \mathrm{N}_{\mathrm{Y}}$ : Relación molar de $\mathrm{X}$ e $\mathrm{Y}$ en el vapor o bien:

$$
\frac{W_{X}}{W_{T}}=\frac{P_{X} M_{X}}{P_{T} \bar{M}_{T}}
$$

Siendo:

$\mathrm{W}_{\mathrm{X}} / \mathrm{W}_{\mathrm{\gamma}}$ : Relación de pesos de $\mathrm{X}$ e $\mathrm{Y}$ en el vapor

$\mathrm{M}_{\mathrm{X}}$ : Peso molecular de $\mathrm{X}$

$\mathrm{M}_{\mathrm{Y}}$ : Peso molecular de $\mathrm{Y}$

De la ecuación 26, se puede decir que en la destilación de una mezcla de dos líquidos no miscibles, las cantidades relativas en peso de los dos líquidos que se recogen son directamente proporcionales a las presiones de vapor de los líquidos a la temperatura de destilación y a sus pesos moleculares. Además, la mezcla destilará a una temperatura constante en tanto exista por lo menos algo de cada uno de los componentes.

\section{Control de calidad.}

Las farmacopeas prevén diferentes ensayos para el análisis de los aceites esenciales, entre ellos son considerados los siguientes:

Determinaciones físicas. Aroma, peso específico, índice de refracción, desviación óptica (poder rotatorio), solubilidad en mezclas alcohol-agua (alcoholes rebajados).

Determinaciones quimicas. Índice de acidez libre, Índices de saponificación y éster. En la determinación de aldehídos y cetonas: formación de fenilhidrazonas, formación de oximas, formación de semicarbazonas, método del bisulfito, indice de acetilo, técnicas cromatográficas, métodos espectroscópicos. Cromatografía de Gases (CG), la cromatografía de capa fina (CCF) se puede utilizar de forma rutinaria para el control de calidad. El uso de la CG como técnica para el control de calidad requiere estándares certificados que permitan hacer una comparación con el producto obtenido y así establecer la calidad del mismo.

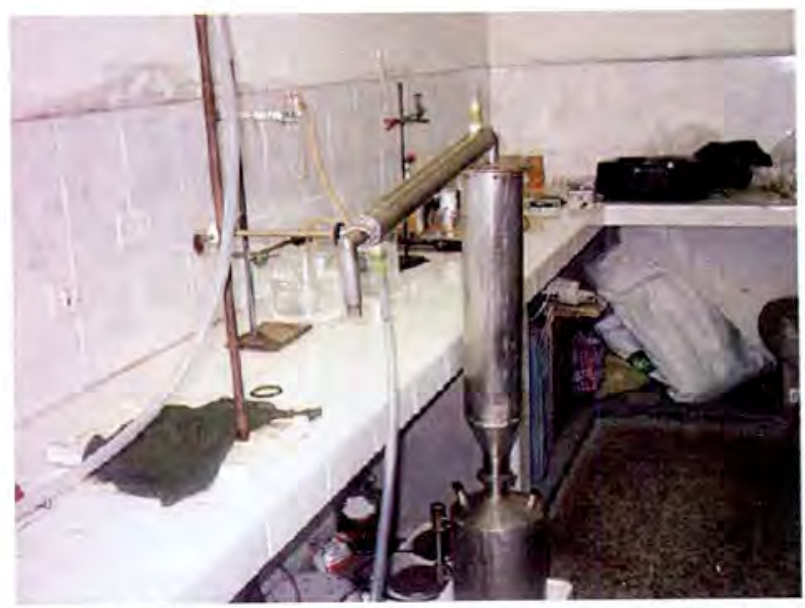

Figura $\mathrm{N}^{\circ} 01$. Equipo extractor por arrastre de vapor ensamblado.

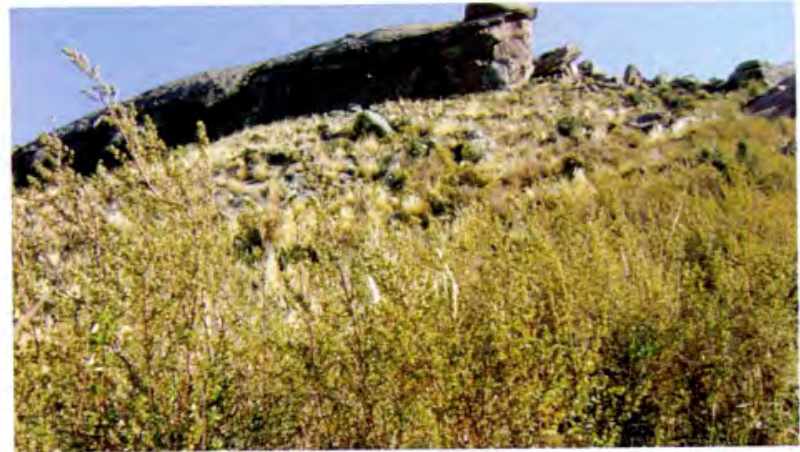

Figura $\mathrm{N}^{\circ} 02$. Muña variedad (Clinopodium bolivianum) Jahilluwaya e Hichu - Puno.

\section{RESULTADOS}

Se presenta a continuación una descripción detallada de las doce corridas experimentales realizadas en este estudio. Cada una de ellas se caracterizó por la operación batch del sistema, salvo en la primera corrida (arranque inicial del sistema), cuyo objetivo fue determinar las condiciones de operación más adecuadas. Se presentan, además, los principales resultados que se desprenden de 
cada corrida experimental, y las conclusiones de las corridas en operación por lote.

\section{Diseño Experimental}

Se estableció un conjunto de 12 pruebas, sobre la base de una matriz de experimentos de $3 \times 4$, tomando la porosidad del lecho y el caudal del vapor, parámetros del proceso se muestran en la Tabla $\mathrm{N}^{\circ} 01$.

\section{Tabla $\mathrm{N}^{\circ} 01$. Matriz de experimentos}

\begin{tabular}{cccccc}
\hline & & \multicolumn{4}{c}{$\mathrm{Q}(\mathrm{ml} / 2.2 \mathrm{~s})$} \\
$\varepsilon$ & 0,0962 & P9 & P10 & P11 & P12 \\
$\varepsilon$ & 0,1154 & P1 & P2 & P3 & P4 \\
$\varepsilon$ & 0,1346 & P5 & P6 & P7 & P8
\end{tabular}

Fuente: elaboración propia

En esta tabla, se muestra el caudal del agua floral por volumetría, en vez del vapor saturado, porque es más preciso y tiene menores fluctuaciones, debido a la condensación del vapor en las conexiones del hidrodestilador.

\section{Resultados de caracterización del aceite esencial de muña}

De acuerdo al protocolo presentado, se lograron resultados como: índice de refracción, potencial de hidrogeniones, polaridad o ángulo de rotación y su densidad respectivamente.

Los resultados se obtuvieron por observación directa de los instrumentos como: refractómetro, potenciómetro, polarímetro y picnómetro respectivamente.

Tabla No02. Características Físicas del aceite Esencial de muña (Clinopodium bolivianum).

\begin{tabular}{ll}
\hline Características Físicas & Resultado \\
\hline 1. Caracteristicas Organolépticas & \\
- Olor & Aromático \\
- Color & Amarillo claro \\
- Aspecto & Ardiente \\
- Estado físico & Líquido \\
2. Índice de refracción & Líquido \\
3. pH & $1,501-1,550$ \\
4. Ángulo de rotación & 5,12 \\
5. Densidad & $29^{\circ} 24$ \\
\hline Fuente: elaboración propia & $0,955 \mathrm{~g} / \mathrm{ml}$ \\
\hline
\end{tabular}

3. Resultados de la extracción del aceite esencial de muña, obtenidos de una muestra de $700 \mathrm{~g}$, se extrae 12,05 $\mathrm{ml}$ en un tiempo de una hora aproximadamente. (ver la Tabla $\left.\mathrm{N}^{\circ} 03\right)$.

La Tabla $\mathrm{N}^{\circ} 03$ muestra los resultados de las 12 corridas por lote, las cuatro primeras corresponden a $700 \mathrm{~g}$ de muestra, cada una en un tiempo de una hora.

4. Resultados del análisis reológico del aceite esencial crudo de muña (Clinopodium bolivianum), los resultados del análisis reológico, fueron obtenidos mediante el software Reocal32 que está incorporada en el viscosimetro Brookfiel,
Málaga V., Análisis de la difusión del aceite esencial de muña (Clinopodium bolivianum) en vapor de agua

Tabla $N^{\circ} 03$. Resultados de la extracción del aceite esencial de muña de parámetros físicos.

Muestra Densidad $(\mathrm{g} / \mathrm{ml}) \quad \%$ Rendimiento Índice refracción $(\alpha)$

\begin{tabular}{llll}
\hline 1 & 0,9551 & 1,569 & 1,550 \\
2 & 0,9463 & 1,509 & 1,508 \\
3 & 0,9381 & 0,989 & 1,509 \\
4 & 0,9363 & 0,991 & 1,520 \\
5 & 0,9321 & 1,347 & 1,501 \\
6 & 0,9361 & 1,486 & 1,516 \\
7 & 0,9550 & 1,517 & 1,506 \\
8 & 0,9549 & 1,520 & 1,524 \\
9 & 0,9371 & 0,987 & 1,515 \\
10 & 0,9509 & 1,347 & 1,524 \\
11 & 0,9550 & 1,517 & 1,540 \\
12 & 0,9540 & 1,569 & 1,550 \\
\hline
\end{tabular}

Fuente: Elaborado por el investigador.

luego de ajustar los datos a diferentes modelos reológicos, el modelo que tiene más significancia estadística, por encima del $95 \%$ de aproximación es el modelo CASSON cuyo modelo matemático es el siguiente:

$$
\sqrt{\tau}=\sqrt{\tau_{y}}+\mu_{\infty} \cdot \sqrt{D}
$$

Siendo:

$\tau$ : Esfuerzo cortante $(\mathrm{Pa})$

$\tau_{y}$ : Esfuerzo umbral $(\mathrm{Pa})$

d: Velocidad de deformación ( $\mathrm{s}^{-1}$ )

$\mu$ : Viscosidad plástica $\left(C_{\mathrm{p}}\right)$

Se aplica para aquellos fluidos en los que no existe proporcionalidad entre el esfuerzo y la velocidad.

\section{DISCUSIÓN DE RESULTADOS}

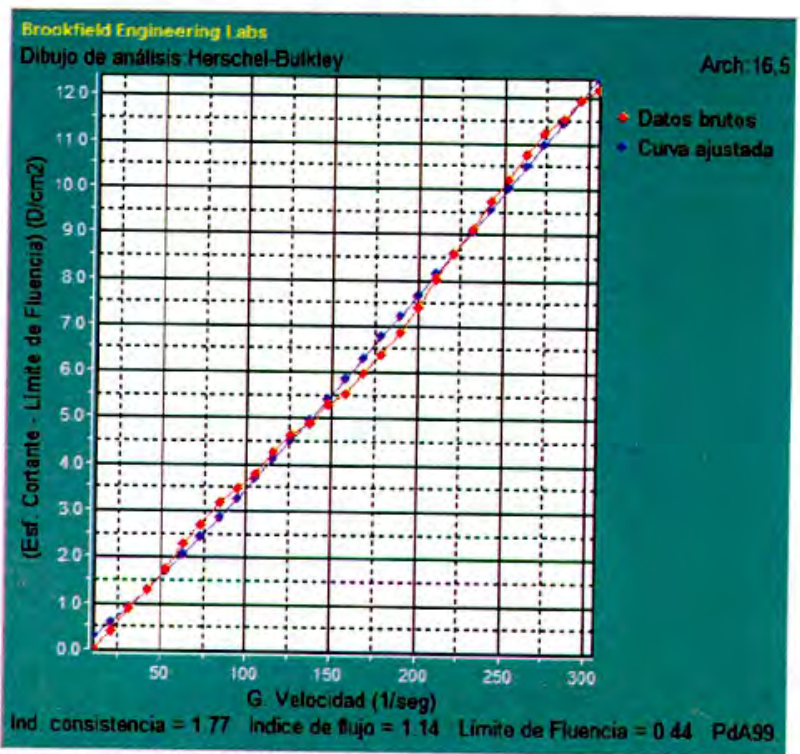

Figura $\mathrm{N}^{\circ} 03$. Variacion del esfuerzo cortante Vs G. Velocidad

Fuente: elaboración con software Reocal32 
En la Figura $\mathrm{N}^{\circ} 03$, se observa el comportamiento reológico del aceite esencial de muña a $16,0^{\circ} \mathrm{C}$, donde los datos brutos son representados por los puntos rojos y los datos ajustados al modelo Herschel Bulkley son representados por las líneas azules. El modelo arroja datos significativos como: índice de consistencia 1,77 Cp, índice de flujo 1,14 , límite de fluencia $0,44 \mathrm{D} / \mathrm{cm}^{2}$, porcentaje de aproximación y significancia del modelo $99,7 \%$.

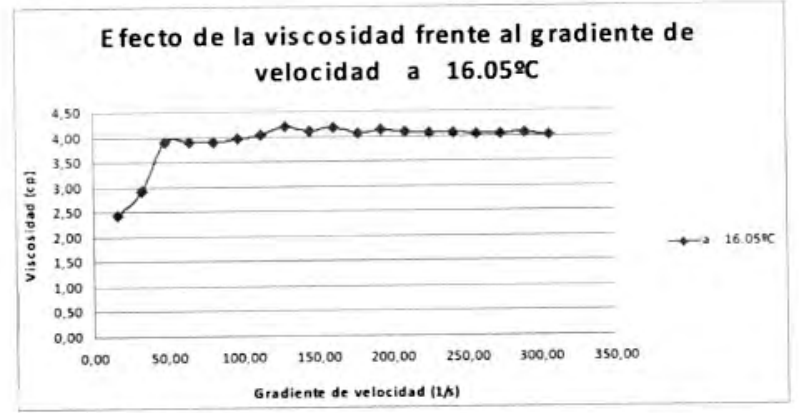

Figura $\mathrm{N}^{\circ} 04$. Efecto de velocidad frente al gradiente Fuente: elaboración propia

En la Figura $\mathrm{N}^{\circ} 04$, se observa el efecto de la viscosidad frente al gradiente de velocidad del aceite esencial de muña, a una temperatura de $16^{\circ} \mathrm{C}$, el comportamiento a menor gradiente de velocidad podemos visualizar menor viscosidad en el punto $(48 ; 2,48)$, con el incremento de la gradiente de velocidad se observa que la viscosidad aumenta de manera relevante, esto ocurre en el punto $(52 ; 3,98)$, finalmente observamos un periodo de estabilización a lo largo de 4,00 Cp y una gradiente de velocidad de $300 \mathrm{~s}$.

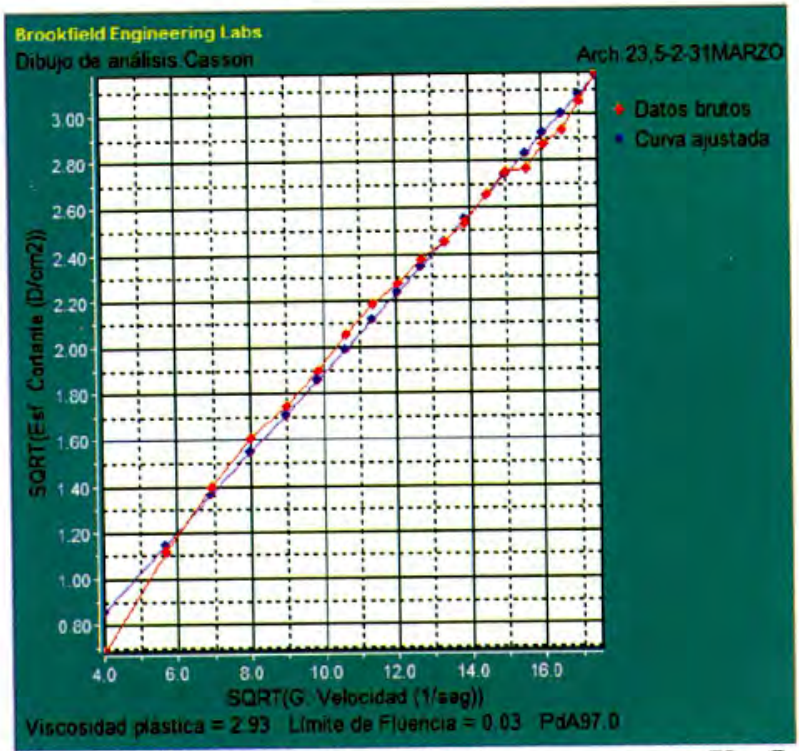

Figura $\mathrm{N}^{\circ} 05$. Variación del esfuerzo cortante Vs G. Velocidad

Fuente: elaboración con software Reocal32

En la Figura $\mathrm{N}^{\circ} 05$, se observa el comportamiento del aceite esencial de muña a $23^{\circ} \mathrm{C}$. Los puntos rojos representan los datos brutos obtenidos, mientras que los puntos azules representan el ajuste de puntos al modelo más adecuado que en este caso, es el modelo de CASSON. Los datos obtenidos de este modelo son los siguiente: viscosidad plástica $2,93 \mathrm{Cp}$, límite de fluencia $0,03 \mathrm{y}$ el porcentaje de significancia o aproximación es de $97,0 \%$.

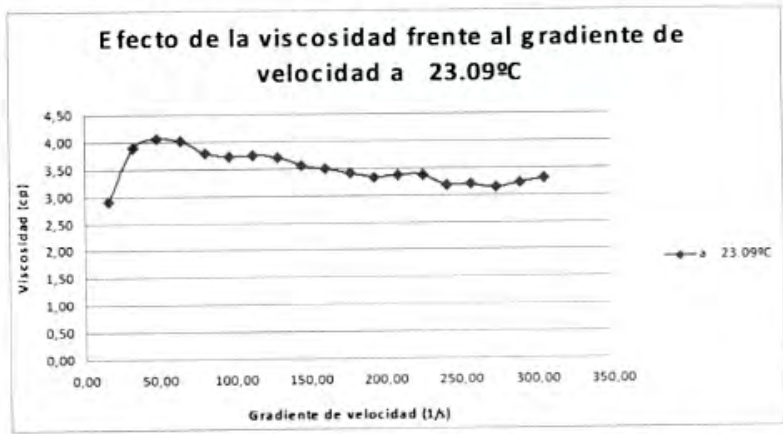

Figura $\mathrm{N}^{\circ} 06$. Efecto de la velocidad frente a gradiente Fuente: elaboración Excel de data de viscosímetro de Brookfield

En la Figura $\mathrm{N}^{\circ} 06$, observamos el efecto de la viscosidad frente al gradiente de velocidad a $23^{\circ} \mathrm{C}$, como en los casos anteriores observamos que existen varios puntos de inflexión. Entre ellos el punto $(50,00 ; 3,90),(60,00 ; 4,04)$ y $(75,00 ; 4,00)$. Posteriormente observamos que la viscosidad tiene una tendencia a bajar a partir del punto $(90,0 ; 3,75)$, hasta el punto $(240 ; 3,25)$ y finalmente en el intervalo de 250 a $300 \mathrm{~s}$ mantiene cierta estabilidad en la viscosidad de 3,20 Cp.

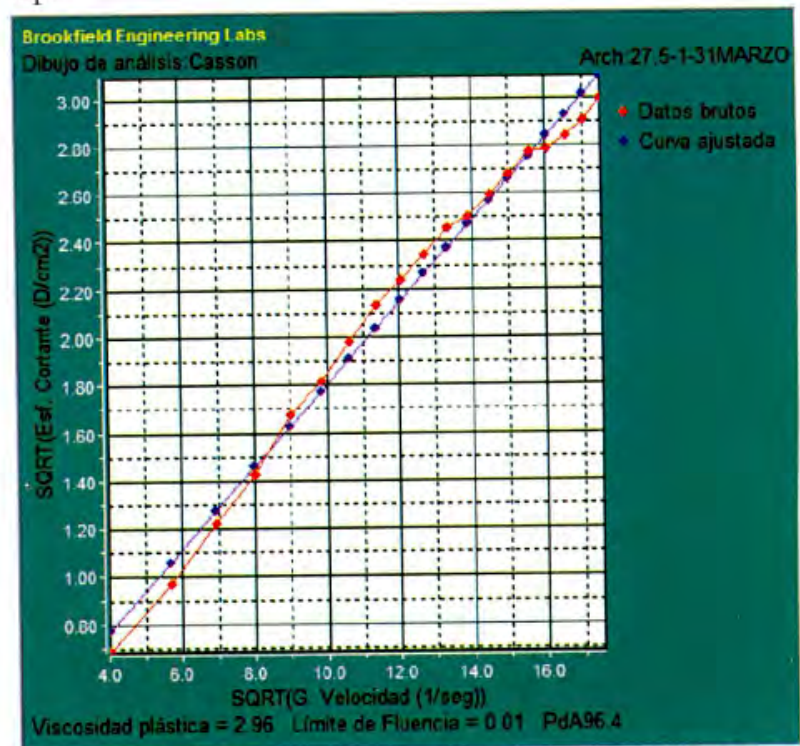

Figura $\mathrm{N}^{\circ} 07$. Variación del esfuerzo cortante Vs G. de Velocidad

Fuente: elaboración con software Reocal32

En la Figura $\mathrm{N}^{\circ} 07$, observamos la variación del esfuerzo cortante versus la gradiente de velocidad a una temperatura de $26,6^{\circ} \mathrm{C}$, donde los puntos rojos representan los datos en bruto, los puntos en azul representan datos de ajuste al mejor modelo encontrado. En este caso atribuimos al modelo CASSON. Como resultado de este modelo tenemos los siguientes datos: viscosidad plástica $2,96 \mathrm{Cp}$; límite de fluencia $0,01 \mathrm{D} / \mathrm{cm}^{2}$ y el porcentaje de aproximación del modelo es de $96,4 \%$.

En la Figura $\mathrm{N}^{\circ} 08$, se observa el efecto de la viscosidad frente a la gradiente de velocidad a $26,6^{\circ} \mathrm{C}$, de 


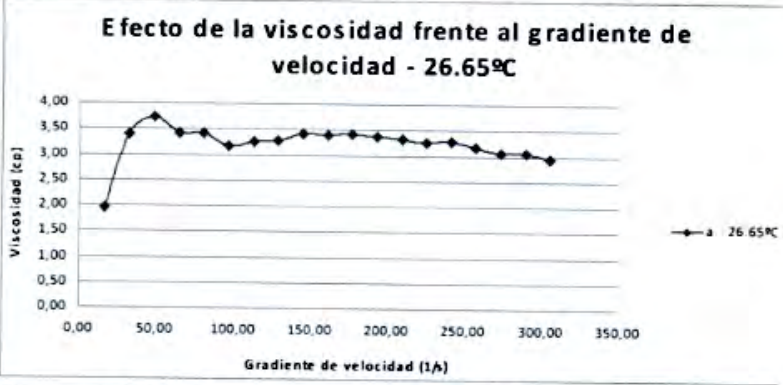

Figura $\mathrm{N}^{\circ} 08$. Efecto de la velocidad frente al gradiente de velocidad

Fuente: elaboración Excel de data de viscosímetro de Brookfield

donde se desprende que la variación de la viscosidad tiende a bajar, a mayor gradiente de velocidad. Inicialmente observamos oscilaciones con punto de inflexión que luego alcanza cierta estabilidad en el intervalo de 150,0 y $200 \mathrm{~s}$. y, finalmente, la tendencia a la baja de viscosidad es evidente, más allá de $250 \mathrm{~s}$.

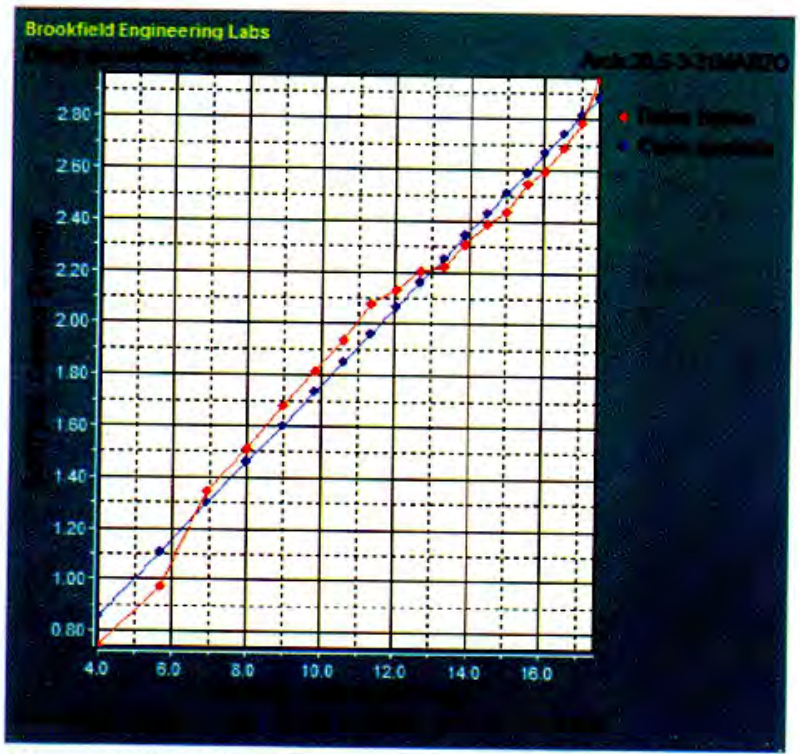

Figura $\mathrm{N}^{\circ} 09$. Variación del esfuerzo cortante Vs G. de Velocidad

En la Figura $\mathrm{N}^{\circ} 09$, observamos el comportamiento del aceite esencial de muña a una temperatura de $30,2^{\circ} \mathrm{C}$, expuesto al análisis de esfuerzo cortante versus gradiente de velocidad, la línea roja representan datos brutos de obtenidos en el experimento, y la línea azul representa datos ajustados empleando el mejor modelo matemático seleccionado que en este caso vendría a ser el modelo CASSON. Los datos obtenidos de este modelo son los siguientes: viscosidad plástica $2,26 \mathrm{Cp}$; límite de fluencia $0,06 \mathrm{D} / \mathrm{cm}^{2} \mathrm{y}$, finalmente, tenemos el porcentaje de aproximación del modelo que es de $95,9 \%$.

En la Figura $N^{\circ} 10$, nos indica el efecto de la viscosidad frente al gradiente de velocidad a la temperatura de $30,2^{\circ} \mathrm{C}$. Al inicio se observa una disminución de la viscosidad de 3,40 a 2,90. Luego hay un incremento creciente en la viscosidad que alcanza hasta $3,75 \mathrm{Cp}$, finalmente, observamos que hay una disminución gradual de la viscosidad, hasta alcanzar estabilidad en el punto $2,60 \mathrm{Cp} y$
Málaga V... Analisis de la difusión del aceite esencial de muña (Cinopodium bolivianum) en vapor de agu

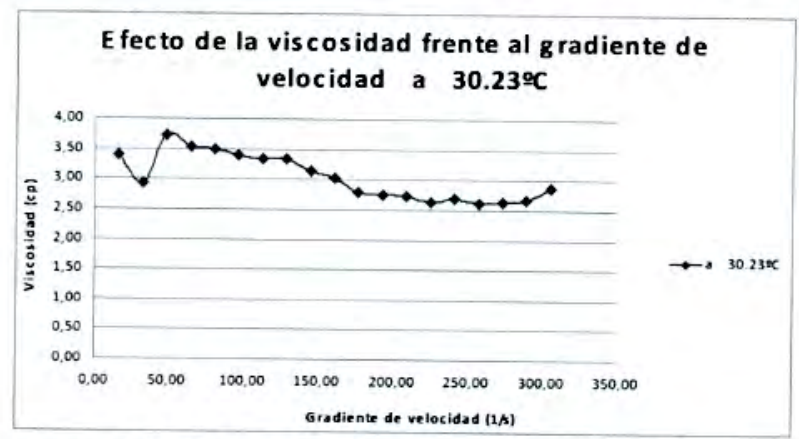

Figura $\mathrm{N}^{\circ} 10$. Efecto de la velocidad frente al gradiente de la velocidad a $30,20^{\circ} \mathrm{C}$

Fuente: elaboración Excel de data de viscosímetro de Brookfield

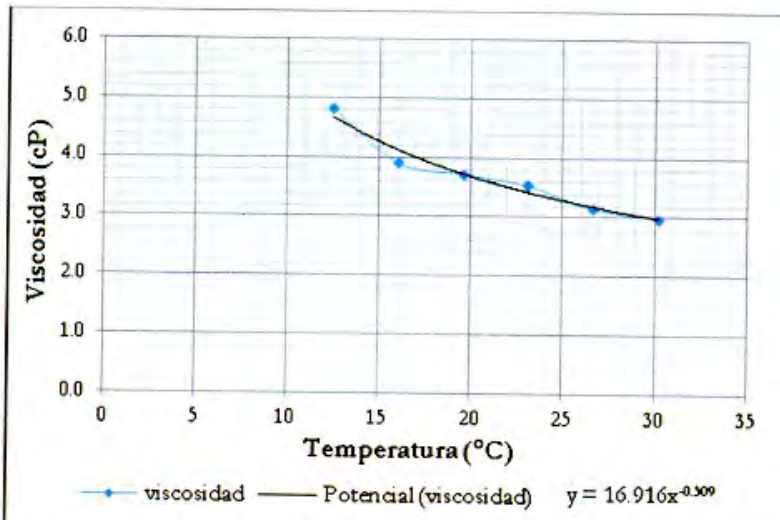

Figura $N^{\circ} 11$. Efecto de la viscosidad frente a la temperatura

Fuente: Elaboración propia en Excel de data de viscosimetro de Brookfield

\section{en el intervalo $225 \mathrm{a} 300 \mathrm{~s}$}

En la Figura $N^{\circ} 11$, se muestra el comportamiento típico de la viscosidad en función a la temperatura. A medida que transcurre el tiempo, la temperatura incrementa. La viscosidad desciende exponencialmente, significa entonces que a mayor temperatura menos viscosidad en el análisis de viscosidad del aceite esencial del crudo de muña (Satureja boliviana).

A temperatura de $12,8^{\circ} \mathrm{C}$ y $4,8 \mathrm{cP}$ se inicia el análisis de la viscosidad, descendiendo esta paulatinamente, a $16^{\circ} \mathrm{C}$ con una viscosidad de $3,8 \mathrm{cP}$, teniendo finalmente una temperatura de $30^{\circ} \mathrm{C}$ una viscosidad de $2,8 \mathrm{cP}$.

\section{Caracterización de la materia prima (Muña) por micro histología}

Después de la hidrodestilación, se observa que los tricomas peltados disminuyeron su volumen o implosionaron (Figura $\mathrm{N}^{\circ} 14$ a). Esto es debido a que liberaron el aceite esencial contenido, conforme fueron envueltos por el vapor caliente. Esta deducción coincide con las publicadas en un estudio previo (Iriti et al., 2006), basado en imágenes por SEM de la lavanda inglesa (lavándula angustifolia). En la observación superficial realizada con imágenes adicionales, no se encontraron tricomas peltados intactos. En el caso de usar la hidrodestilación asistida por micro-ondas (MAHD o MASD) para la lavanda inglesa (lavandula angustifolia), los resultados publicados son diferentes (Iriti et al., 2006). Los tricomas glandulares poseen 
agujeros o han explotado completamente, debido a exposición al micro-onda. Cuando se usa la extracción por fluidos supercríticos para obtener aceites esenciales, en el caso del origanum virens (Gaspar et al., 2001), se ha demostrado que los tricomas glandulares explotan durante la expansión del fluido supercrítico, debido al gradiente de

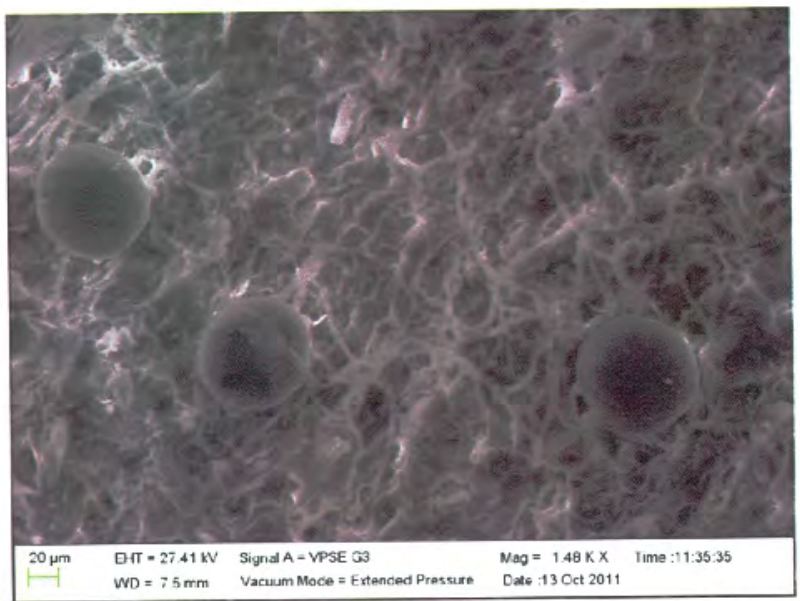

presión existente entre el aceite $+\mathrm{sc}-\mathrm{CO}_{2}$ contenidos y el exterior.

Este análisis micro-histológico permitió determinar la eficiencia del contacto entre el agua y la matriz herbácea, al no detectarse tricomas intactos, la abundancia y ubicación asimétrica de los tricomas glandulares, la ausencia

Figura N¹2 a. Imagen por SEM de la superficie de la hoja de muña antes del proceso: Con 897 X y una escala de $100 \mu \mathrm{m}$. Y $1480 \mathrm{X}$ y una escala de $20 \mu \mathrm{m}$.
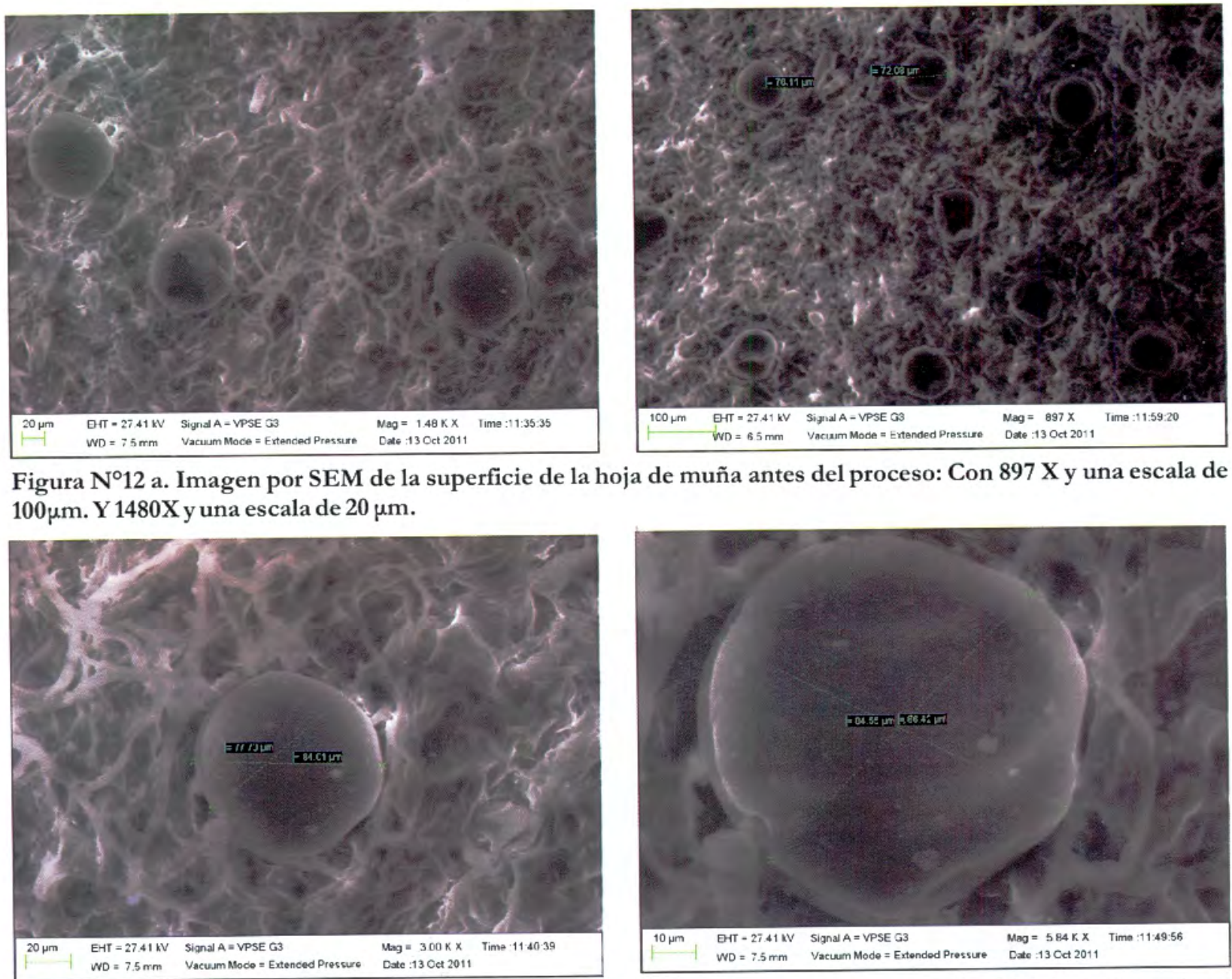

Figura $N^{\circ} 13$ a. Imagen por SEM de la superficie de la hoja de muña antes del proceso: Con $3000 \mathrm{X}$ y una escala de $20 \mu \mathrm{m}$. Y $5840 \mathrm{X}$ y una escala de $10 \mu \mathrm{m}$.
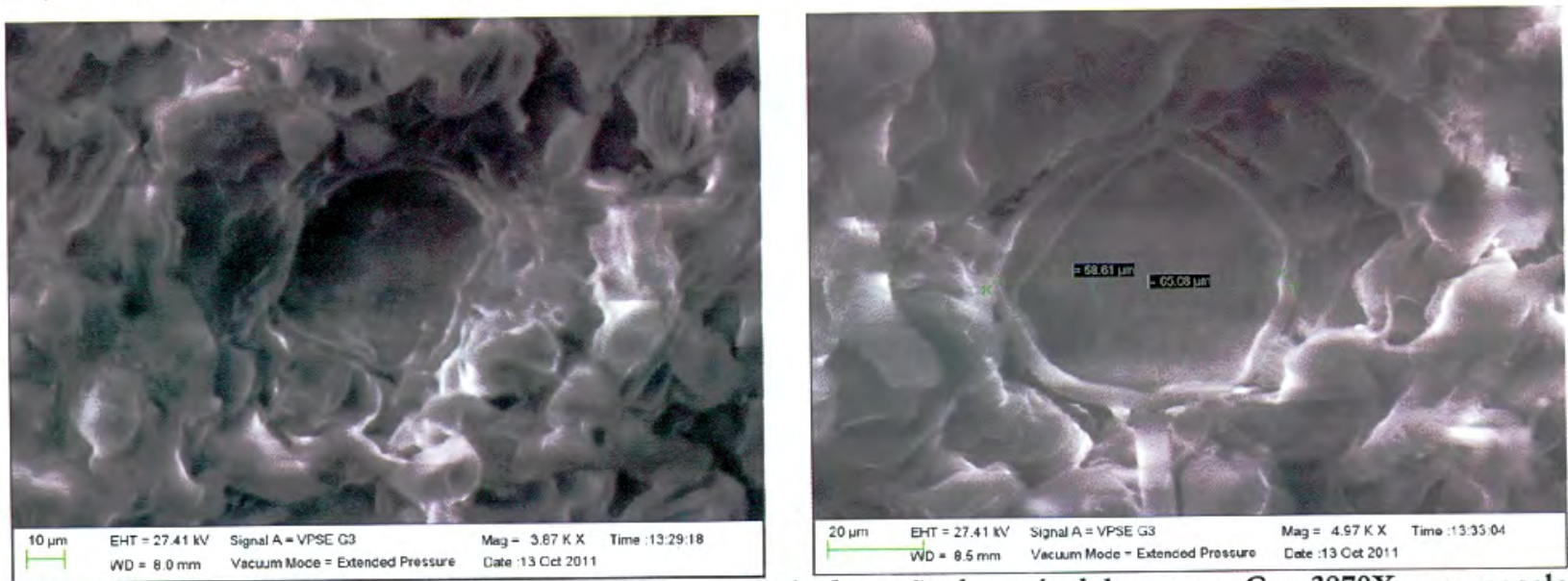

Figura $\mathrm{N}^{\circ} 14$ a. Imagen por SEM de la superficie de la hoja de muña después del proceso: Con 3870X y una escala de $10 \mu \mathrm{m}$ y Con $4970 \mathrm{X}$ y una escala de $20 \mu \mathrm{m}$. 
de poros en la superficie de la matriz, lo cual descarta la hipótesis de una difusión del vapor saturado a través de ella. Al comparar las imágenes por SEM y las deducciones establecidas, con las publicadas para otros procesos de obtención de aceites esenciales, se establece que el fenómeno de liberación del aceite esencial desde los tricomas glandulares (exudación), tiene una influencia dominante del aumento de la temperatura y del tiempo de exposición de la matriz herbácea a la corriente de vapor de agua. Además, este fenómeno es más lento que el analizado para los otros procesos de obtención, porque la cuticula no sufre daño físico superficial; por lo cual, el aceite es liberado paulatinamente, durante un tiempo mayor al observado para los otros procesos.

Este análisis constituye una herramienta importante para formular modelos fenomenológicos microscópicos más próximos a la realidad, que tomen en cuenta la verdadera morfología de la matriz herbácea, la localización real del aceite esencial en ella y conocer la variación de la morfología superficial de la matriz, después del proceso. Más adelante, se formula un modelo fenomenológico básico, usando estas deducciones.

\section{CONCLUSIONES}

Según el trabajo desarrollado podemos concluir que el aceite esencial de muña fue obtenido con un rendimiento de $1,362 \%$ de hojas y tallos en promedio de 12 pruebas experimentales, a nivel bench, con caudales de refrigeración constante y porosidades del lecho variable. $\mathcal{E}=0,0962 ; \varepsilon=0,1154$ y $\varepsilon=0,1346$ señalados en la Tabla $\mathrm{N}^{\circ} 01$.

La reología del aceite esencial de muña es de tipo viscoplástico correspondiente al modelo de CASSON, operado por el viscosímetro Brookfiel modelo DV III ULTRA, cuyo resultado de viscosidad es de $3,8 \mathrm{Cp}$ a $13^{\circ} \mathrm{C}$ y $2,8 \mathrm{Cp}$ a $30^{\circ} \mathrm{C}$.

El aceite esencial está situado en los tricomas glandulares de la superficie de las hojas de la muña distribuidos asimétricamente en el anverso y reverso de las hojas. En contacto con el vapor saturado, los tricomas liberan el aceite esencial contenido e implosiones, disminuyendo su volumen y cambiando su forma. Se detectaron poros en la superficie de las hojas, lo que indicaría la difusión del vapor en el interior de la hoja con un diámetro promedio de $84,55 \mu \mathrm{m}$.

El modelo matemático predice la evolución de la concentración de aceite esencial de lecho cargado de muña en cada intervalo de tiempo, así mismo los parámetros del modelo, obtenidos por tratamiento de datos experimentales predice la difusividad efectiva que en promedio es: $D_{\text {eff }}=1,69$ $\mathrm{x} 10^{-5} \mathrm{Cm}^{2} / \mathrm{s}$, los cuales sirven para comparar la velocidad de difusión de este aceite frente a otros casos y el área de transferencia de masa efectiva existente en la matriz herbácea al inicio y durante el proceso. válaga V., Anàlisis de la difusión del aceite esencial de mutia (Clinopodium bolivianum) en vapor de agua

\section{REFERENCIAS BIBLIOGRÁFICAS}

Benyoussef, E.-H.; Hasni, S.; Belabbes, R.; Bessiere, J.-M. Modélisation du transfert de matiére lors de l'extraction de l'huile essentielle des fruits de coriandre. Chem. Eng.J. 2002, 85, 1-5.

Bezdolny, N.I. and Kostylev, V.P. Plant for Processing Vegetal Stock. U.S. Patent 5,372,680, Dec. 13, 1994.

Boucard, G.R. and Serth, R.W. Practical Design of a Continuous Distillation Plant for the Separation of Essential Oils from Aromatic Raw Materials. Technical Report; Texarome Inc.: Texas, USA, 2005.

Brat, P. Ollé, D. Gancel, A-L. Reynes, M. Brillouet, J-M. Essential oils obtained by flash vacuum-expansion of peels from lemon, sweet orange, mandarin and grapefruit. Fruits, 2001, 56, 395-402.

Cerpa, M.G. y Cocero, M.J. Comparación entre los aceites esenciales hidrodestilados y los extractos obtenidos con altas presiones. Cartel presentado a la $2^{a}$ Reunión de Expertos en Tecnologías de Fluidos Comprimidos. Valladolid, Octubre del 2005.

Chemat, F.; Lucchesi, M.E.; Smadja, J.; Favretto, L.; Colnaghi, G.; Visinoni, F. Microwave accelerated steam distillation of essential oil from lavender. Analytica Chimica Acta, 2006, 555, 157-160.

Churchill, S.W. and Chu, H.H.S. Correlating equations for laminar and turbulent free convection from a vertical plate. Int. J. Heat Mass Transfer. 18, 1323-1329, 1975.

Cocero, M.J. Procesos comerciales de extracción con fluidos supercriticos. Ingenieria Quimica. 2006. N ${ }^{\circ} 441$, Noviembre, 144 157. Code of Federal Regulations. U.S. Government Printing Office. Title 21, Vol. 3, Chapter I, Part 182, Subpart A, 456-460. April 1,2003.

Coutiere, D. Process for the Continuous Hydro-Distillation of Plants. U.S. Patent 4, 935, 104, Jun. 19, 1990. Del Valle, J.M.; De la Fuente, J.C.; Cardarelli, D.A.J. of Food Eny. 2005, 67, 35-37.

Essential Oils. Kirk-Othmer Encyclopedia of Chemical Technology, 4Th Ed.; Wiley \& Sons. New York, USA, 1993; Vol. 17,pp 1-60.

Ferhat, M.A.; Meklati, B.Y.; Smadja, J.; Chemat, F. An improved microwave Clevenger apparatus for distillation of essential oils from orange peel. Journal of Chromatography A, 112, 2006, 121-126.

Gallego, L.C. y Cardona, C.A. Aplicaciones industriales de los fluidos supercríticos (I): Generalidades y procesos de extracción. Ingenieria Quimica. 2004

Gersbach, P.V. The essential oil secretory structures of Prostanthera ovalifolia (Lamiaceae). Annals of Botany. 2002, 89:255-260.

Heath, H.B. and Reineccius, G. 1986. Flavor Chemistry and Tecbnology. AVI Publishers, USA. Hidrodestilación de aceites esenciales: Modelado y Caracterización Manuel G. Cerpa 1-39.

Iriti, M.; Colnaghi, G.; Chemat, F.; Smadja, J.; Faoro, F.; Visinoni, F.A. Flavour Fragr: J. 2006; 21:704-712.

Muñoz, F. Plantas medicinales y aromáticas: Estudio, cultivo y procesado. $4^{\mathrm{a}}$ Reimpresión. Ediciones Mundi-Prensa: Madrid, España, 2002.

Palomino, A. y Cerpa, M. Hidroextracción de los aceites esenciales. Memorias de la IV Reunión de Fenómenos de Transporte. Callao, Perú, 1999 .

Peter, K.V. Handbook of Herbs and Spices. Woodhead Publishing Limited: London, England, 2004.

\section{Correspondencia:}

Vicente Málaga Apaza: malagavice@hotmail.com
Fecha de Recepción: 27/06/2014

Fecha de Aceptación: 09/07/2014 